\title{
PROTECTION DESIGNS IN MEDIUM VOLTAGE DISTRIBUTION NETWORKS, TIME TO RECONSIDER
}

\author{
Gerard Schoonenberg \\ Jan Stoelhorst \\ Alex Pikkert \\ Eaton Holec, Medium Voltage \\ Hengelo, The Netherlands \\ holec-info@eaton.com
}

\section{INTRODUCTION}

The quality of the electricity supply has always been a point of major interest. Worldwide there is an increasing concern about Power Quality [1,2].

On the other hand the electricity-market is quite conservative. This implies that long used solutions are often not questioned, so in general no reconsideration takes place anymore, although more economical or technical optimised solutions may exist. In addition to this the technical know-how in the utilities nowadays seems subsidiary to the (short term) economical concerns.

This article explains what the real backgrounds are for the widely used fuse protection of transformers. The fuses dealt with are considered to be of the current limiting type, as mainly used in Europe; so expulsion fuses are not taken into account. With the help of a few examples it is demonstrated that a lot of misinterpretations exist about application aspects.

After a historical overview of $M V$-transformer protection in the Netherlands, as applicable for many other countries, the nowadays considerations for this protection are dealt with. This results in listing the pros and cons of protection with fuses compared to circuit-breakers (CB's).

The conclusions of these considerations were used for the design and main specifications of a new generation of indoor switchgear for 12 and $24 k V$ networks. (Fig.1) [3].

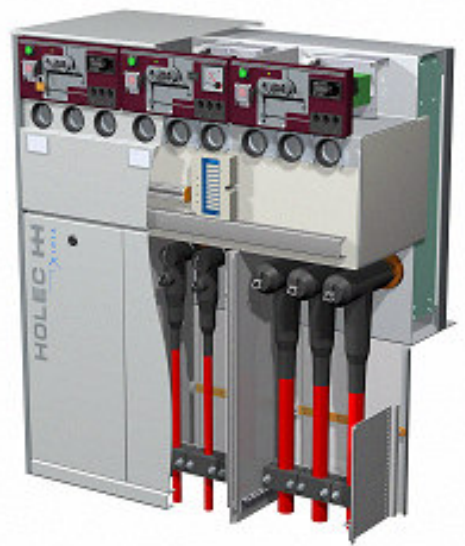

Figure 1 24kV Ring Main Unit Xiria (Eaton Holec)

\section{PROTECTION OF MV TRANSFORMERS IN THE NETHERLANDS, OVER THE YEARS}

\section{First generation (up till 1950): CB's}

In the past (app. 1950) distribution transformers were protected against overload and short-circuit by means of CB's with overcurrent relays consisting of a thermal element and an instantaneous high set over current element. The thermal element provided the overload protection, the instantaneous high set over current element provided the short-circuit protection.

A transformer was installed including the corresponding $\mathrm{CB}$ and protection and this fixed setting was never looked after again.

As the number of the connected consumers grew with everincreasing load, there was no idea of this load increase. At an undefined moment the transformer was switched off by the overload protection. At that moment the existing transformer had to be changed for one with a larger rating or another transformer had to be added.

\section{Second generation (>1950): CB's and current limiting fuses}

After the first generation protection, the maximum load of the transformer was measured with maximum demand ammeters. These meters gave on a base of 8 or 15 minutes time delay a kind of average value of the current. The highest measured value was stored by means of the red slave pointer in the ammeter. After reading this value for example once a year, the slave pointer was reset and a new registration was started. The obtained measured values gave the trend in the maximum load of the transformer over the years. It could be predicted when the transformer would be overloaded and should be replaced. In practice it was not likely that a transformer would be overloaded. So an overload protection was not necessary.

CB's at the HV-side of transformers were generally provided with definite time overcurrent relays or inverse time overcurrent relays, setting often 1,5 times rated current of the transformer or higher (so no overload protection). Relays with thermal characteristic (overload protection) were placed sometimes in industrial connections, where no protection was placed at the low voltage side of the transformer.

In the mean time the HV current limiting fuse (HRC-fuse) was developed. This introduced a simple and economical solution for open systems, as only 2 clamps were needed to 
have the fuse in its place in the circuit. The advantage of simplicity was lost when the fuse holder had to be integrated in encapsulated switchgear.

\section{Third generation $(>1960)$ : current limiting fuses encapsulated}

With the introduction of the load-break switch / fuse combination in cast-resin insulated ring main units (like Eaton Holec's Magnefix), there was an economical and compact solution for switching and protection. The fuses as manufactured in that time, only could break currents from about 2 times rated current of the fuse, this is about 4 times the rated current of the transformer. In other words, there was only a short-circuit protection and no overload protection available.

The application of load-break switch / fuse combinations is still widely in use nowadays. However, their application is restricted in $10 \mathrm{kV}$ networks to transformers of maximum $1000 \mathrm{kVA}$ size, because of the restricted range of rated currents of fuses. Some utilities apply load-break switch / fuse combinations for transformers up till 400kVA maximum, because of the selectivity between the fuses and the upstream protection (at the beginning of the - open ring).

The application of fuses at the HV-side of the transformer normally is combined with a low voltage main breaker with overload and short-circuit protection. In distribution networks however, a main CB is generally not applied, but only the outgoing groups are individually protected.

The most commonly used fuse was of the general purpose type. This type has the drawback that the fuse will burst or even explode on overloads (or on normal loads when one or more of the vulnerable fuse-wires are damaged). To overcome this drawback the 'full range type' has been developed in the early 80's of last century. However, the costs are up to twice as high for this type of fuse. The types of fuses are defined in IEC 60282-1 [8].

\section{Fourth generation (>1980): CB's with digital protection relays and full-range fuses:}

(Refer to the paragraph above for the full range fuses.) The evolution to digital protection relays makes life simpler for design engineers, as much smaller size current transformers can be applied nowadays. The reason for this is the large setting range of modern protection relays. Therefore it is possible to use much larger CT-ratios, than necessary in older designs.

However, in defining the current transformer (CT) for the application of a CB as transformer breaker, it is still common practice to start with the conversion of the transformer power into the rated current of the transformer. E.g. for a $400 \mathrm{kVA} / 10 \mathrm{kV}$ transformer this results in a rated current of $23 \mathrm{~A}$ and therefore a CT-ratio of $25 / 5 \mathrm{~A}$ or $25 / 1 \mathrm{~A}$ would be chosen. This is not the right way to do!
The setting range for the overcurrent protection of a modern relay is e.g.: $0,1 \ldots 2,4 \mathrm{x}$ the rated current. The approach should then be as follows: determine the desired current setting of the relay expressed in a current value at the primary side of the current transformer. Determine the desired art of protection: definite time overcurrent, inverse time overcurrent or time overcurrent with thermal curve, with or without an instantaneous (or definite) time overcurrent element for short-circuit protection. Try to make a combination of available current transformers and protection relays which meets the required current setting or which approaches this as near as possible. A relay setting of some times the rated current of the transformer is surely not worse than a fuse! This implies for the above-mentioned $400 \mathrm{kVA}, 10 \mathrm{kV}$ transformer the following (as an example):

The rated current is $23 \mathrm{~A}$. The desired setting is 1,5 times rated current. In combination with the overcurrent relay setting of 0.1 .2 .4 , the lowest possible CT ratio will be 300/5 (or 300/1). This CT has much smaller dimensions and is easier to design than the above-mentioned 25/5 (or 25/1) CT.

\section{Examples of protection schemes}

Consider e.g. a $1000 \mathrm{kVA}$ transformer at $10 \mathrm{kV}$ level. The rated current of this transformer is 57,7 A. Both fuse and relay protections for this transformer are compared.

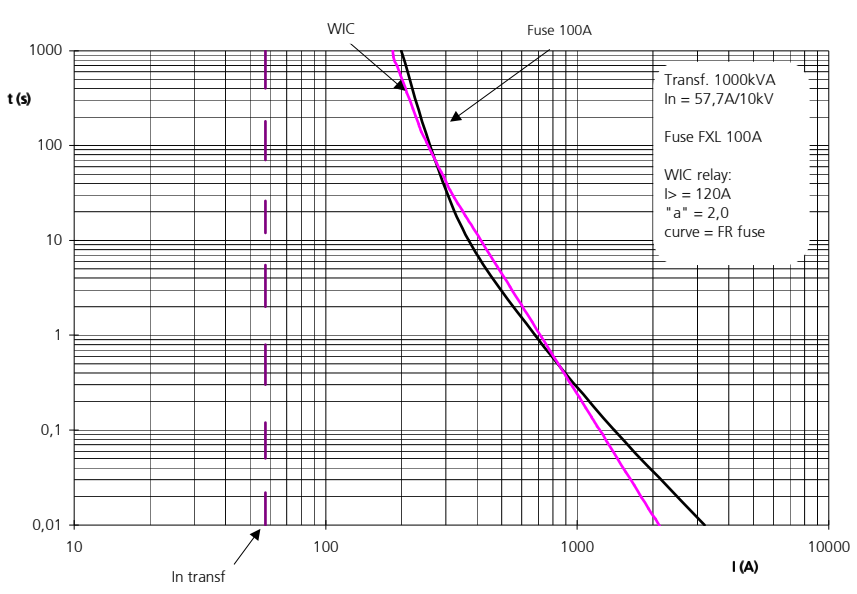

Figure 2 Fuse characteristic compared with relay setting

In fig.2, the current-time characteristic of a fuse Bussmann type FXL*J $100 \mathrm{~A}$ and a CB with a SEG protection relay, type WIC, with setting: I> = 120A; "a" = 2,0; curve = FR fuse are given.

The chosen current setting $I>=120 \mathrm{~A}$ which is 2,1 times the rated current of the transformer just as the time setting "a" $=2,0$ are examples. Settings always must be compared with upstream and downstream protections to achieve selectivity. 


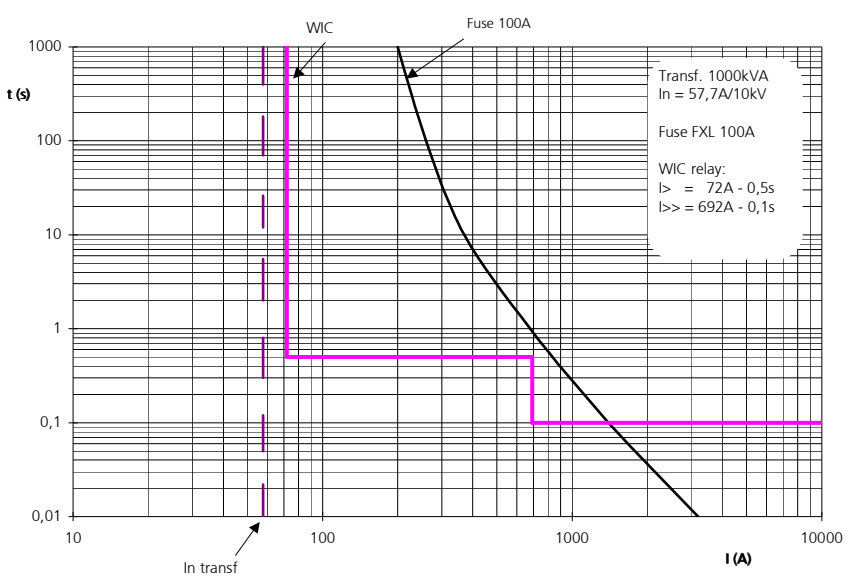

Figure 3 Fuse characteristic compared with relay setting

In fig.3, the same fuse characteristic as in fig. 2 is given and also the SEG type WIC relay, now with setting: $\mathrm{I}>=72 \mathrm{~A}$; $\mathrm{tI}>=0,5 \mathrm{~s} ; \mathrm{I}>>=692 \mathrm{~A} ; \mathrm{tI}>>=0,1 \mathrm{~s}$ as protection for the transformer $1000 \mathrm{kVA}, 10 \mathrm{kV}$.

The chosen current setting $\mathrm{I}>=72 \mathrm{~A}$ which is 1,25 times the rated current of the transformer, the current setting $\mathrm{I}>>=$ $692 \mathrm{~A}$ which is $12 \mathrm{x}$ the rated current of the transformer just as the time settings $\mathrm{tI}>=0,5 \mathrm{~s}$ and $\mathrm{tI}>>=0,1 \mathrm{~s}$ are examples.

It is obvious that with the protection relay/CB combination, far more possibilities to create selectivity and fine tuning are available.

\section{NOWADAYS CONSIDERATIONS ABOUT FUSES AND CIRCUIT-BREAKERS (CB's)}

\section{Fuses [8]:}

- For protection of distribution transformers up to a maximum power rating ( $1 \mathrm{MVA}$ at $10 \mathrm{kV}$ ), the application of current limiting fuses is wide spread. For higher ratings, no suitable fuses exist, so CB's are used.

- In general, so called general purpose fuses are in use. The fuse wires will melt at currents larger than app. 1.2 times the designated rated current of the fuse. Within enclosures, derating must be applied because melting will occur at lower current values. As fuses are in general vulnerable for mechanical stress (impact when placing in switchgear, transport in cars etc.), it is quite possible that an internal wire is already broken, directly after installation. When e.g. one of 4 wires is broken, the melting current will reduce from 1,2 to $1,2 * 0,75=0,9$ times the rated current. General-purpose fuses are in this region of overload currents (and below) not capable of interrupting the current and an explosion is inevitable.

- Another type of fuse, the back-up type, is only meant for interrupting short circuit currents. This type of fuse has an even larger gap between melting current and minimum breaking current, than the general purpose fuse.

- Full range type fuses largely cover the drawback as stated above; these can interrupt every current down to its rated value. However, they are more expensive than general purpose fuses.

\section{Circuit-breakers (CB's):}

- Nowadays the arcing media SF6 and vacuum are the only practical alternatives for newly developed medium voltage CB's. Switching in vacuum is world wide accepted and still growing market share to more than $50 \%$.

- As a consequence of the choice for vacuum, which is maintenance free, the switchgear designs nowadays evolve into fixed breaker designs.

- Vacuum-CB's have a stable current interrupting behaviour. Either the contacts open followed by interruption of all currents, or the contacts stay closed and carry all currents without a problem. With modern protection relays, all kinds of I-t curves van be chosen, so real optimization with upstream and downstream protections is possible.

\section{CB's instead of fuses, the pros:}

There are several developments going on worldwide:

- Increasing load of the electricity grid.

- Widespread use of dispersed generation.

- Introduction of simple, economical vacuum CB's that are maintenance free. With these it is possible to built very compact RMU's like Xiria [3].

- Demand for simple and safe operation.

- Demand for green and clean designs (easy handling at end of life).

- Steadily reducing costs for intelligence, sensors and communication.

A logical step is to replace the relatively bulky and expensive HV fuse by a CB to protect the transformer (Fig.4). This has, amongst other things, the following advantages: $[3,5,6]$

- Extra life cycle costs of fuses with its environmental impact: The energy loss in practice is about 100 times higher for a MV panel with fuses compared to that of a panel with a CB. As an example, consider a $400 \mathrm{kVA}$, $10 \mathrm{kV}$ transformer with its rated current of 23,1 A. The power loss in the applied $25 \mathrm{~A}$ or $31,5 \mathrm{~A}$ fuse will be roughly $100 \mathrm{~W}$ in total for the 3 poles, depending on the cooling possibilities for the fuses. The fuse equals a (hot) resistance of app. $60 \mathrm{~m} \Omega$, where e.g. a complete $\mathrm{CB}$ panel of the Xiria RMU is less than $0,6 \mathrm{~m} \Omega$, so $1 \%$ of the fuse value. As the grid is more and more used to its limits, the maximum dissipation will be reached in many situations. Consider for the time being an average 50W loss for the fused link. This already means an extra dissipation of 440 $\mathrm{kWh}$ per year, which is not present when the transformer is protected with a $\mathrm{CB}$ !

- No limitation in current ratings for the settings of the protection.

- Possibility to be fitted with protection systems, which are independent of external power, with close discrimination with the LV fuses.

- Interruption of all possible currents, including those, characteristic to evolving faults.

- Earth fault protection is possible.

- Possibility to add Buchholz protection, thermal -, etc, without extra costs. 
- With one single combination of CT and protection relay, complete rings can be protected; No stock necessary for various types of fuses, minimizing the making of mistakes.

- With CB's real sealed for life designs are possible. There is no need for access to primary parts, except of course for the cable connection. The medium voltage (live) parts of the switchgear don't have to be touched when changing the settings of the protection or restoring energy after a fault. In this way the design can be very compact. Modern RMU's, like Xiria, imply also maximum safety and environmental design [3].

- Reduced maintenance: the medium voltage (live) internal parts of the switchgear can be fully sealed when there is no need for access to primary parts such as fuses, avoiding deterioration due to moisture and dust. The equipment is expected to have a very high degree of reliability

- No need to travel around with (mechanically) vulnerable HV-fuses.

- Fuses deteriorate over the years, depending on (over)currents. For this reason, they should be checked (resistance measurement) every 10 years. Some users however decided to exchange the fuses every 10 years for new ones, because of the relatively low costs, compared to the costs resulting from a failure of a fuse. After a failure, the fuses in all 3 poles must be replaced. It is assumed that the testing and replacement costs, approximately every 10 years, plus stock and handling costs for the fuses equal the testing costs of the CB.

- MTBF figures are 1000-5000 years, both for fuses and CB's [4]. However, in case of failure, a fuse will always burn, or even explode, where a CB generally does not open.

- Designs with fuses need earthing on both sides of the fuse, being more complex than designs with CB's.

- Suitability for remote restoration of the network with lower costs. Today's switchgear equipment in the lower area of the medium voltage distribution is mainly hand operated Restoring power in the network takes time, depending on the density of the network and the local circumstances regarding long distances or areas inaccessible due to heavy traffic, or due to environmental conditions like snow and ice. Depending on costs, remote control will be more widely used.

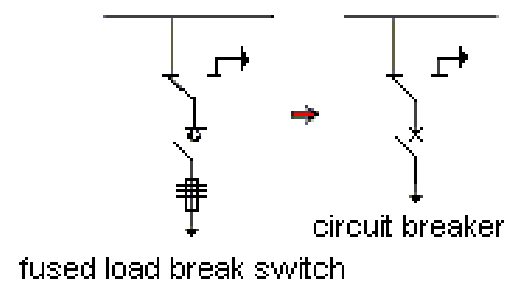

Figure 4 Circuit-breaker and fused load break switch

\section{CB's instead of fuses, the cons:}

- Current limitation: An often-heard drawback of CB's compared to fuses, is that there is no (peak) current limitation. This does not seem to be a point of real concern, since CB's have always been used satisfactorily

to protect larger transformers (above 400 to $1000 \mathrm{kVA}$ ). Fault currents in a transformer start as overload currents, created e.g. by a short circuit between windings at HV or LV side. A fault will develop into a larger short circuit current. Especially with fuses, this development time should not take too long as the fuse might not be able to clear the current. For shorter transition times to a full short current, a CB has generally about $100 \mathrm{~ms}$ to react and clear the current, which should be enough. [7]. Thereupon, the fuse holder itself is an extra possible source for open arcs.

- Simplicity: This sure was the case in open constructions. With the enclosed HV designs nowadays, the simplicity is not a valid argument anymore.

- Costs: A cost comparison between the total life cycle costs, plus the costs resulting from managing fuses versus the company procedures for CB's and the non-availability of the network is already made in the chapter before.

\section{CONCLUSIONS:}

- Nowadays both circuit-breakers and protection devices are developed to such a mature level, both in economical and in technical way, that application for the smaller transformer ratings is also feasible.

- There is no place for fuses in a modern concept of a RMU like Xiria.

- Modern, digital relays create the possibility to apply much smaller current transformers, than would be necessary in the past.

- Vacuum CB's with a self-powered protection relay, offer a solution that is better on a economical basis, but also on most technical aspects.

- The often claimed advantage of current limiting aspect is of minor importance as the switchgear with cables upstream of the fuse are also to be considered, because they are in the same substation.

- A better control of the network is contributing to the quality of the network if the majority of RMUs is equipped with intelligence and communication devices. This will be made possible in the near future because prices of protection and communication devices related to their functionality continue to decrease, as the costs for non delivered energy are steadily increasing.

\section{REFERENCES:}


[4] Bollen, M.H.J., "Betrouwbaarheidsgegevens voor componenten in distributienetten", in Energietechniek, November 1994, P 664-670 (in Dutch)

[5] Blower, R.W. ea., "Trends in distribution transformer protection", in Proceedings 3rd IEE Conference on Future trends in distribution switchgear, London, april 1990. Publication 318. Coden: IECPB4 ISSN:0537-9987. P7-11

[6] Brüggeman, G. ea., "MV/LV distribution transformer fault protection", in Proceedings CIRED conference, Liege, 1991 AIM 1991. P1.14/1-6 vol 1

[7] Elektrotechnisch Zeitschrift Bd. 108 (1987) h.16 (in German)

[8] IEC publication IEC 60282-1: High-voltage fuses part1: current limiting fuses; fith edition, 2002 